\title{
Solitary Necrotic Nodule of the Liver: Different Pathological Findings Express a Different Histogenesis
}

\author{
Rosalia Patti ${ }^{a}$ Daniela Cabibi ${ }^{b}$ Massimiliano Sparacello \\ Gaetano Di Vita ${ }^{a}$ Giuseppe Montalto ${ }^{c}$ \\ Departments of a Surgical and Oncologic Science, ${ }^{b}$ Human Pathology and ${ }^{c} \mathrm{Clinical}$ \\ Medicine and Emerging Pathologies, University of Palermo, Palermo, Italy
}

\section{Key Words}

Solitary necrotic nodule $\cdot$ Histogenesis $\cdot$ Liver tumor $\cdot$ Metastases

\begin{abstract}
Solitary necrotic nodule of the liver is a rare benign lesion the histogenesis of which is still debated. We here report the case of a patient who underwent laparoscopic cholecystectomy for gallstones and who was accidentally found to have a solitary necrotic nodule of $2 \mathrm{~cm}$ in the fifth segment of the liver. On the basis of the histological findings, the hypothesis that different pathogenetic mechanisms could be involved in the histogenesis of this lesion is discussed.
\end{abstract}

\section{Introduction}

Solitary necrotic nodule (SNN) of the liver is a rare benign lesion first reported in 1983 by Shepherd and Lee, who described four lesions with a completely necrotic core and a hyalinized fibrotic capsule containing elastic fibers [1]. To date, fewer than 60 cases have been reported. Koea et al., in a revision of the literature performed up to 2003, reported only 50 cases, the majority of which were found in males and over three-quarters in the right lobe of the liver [2].

We here report a patient who underwent laparoscopic cholecystectomy for multiple lithiasis and who was accidentally found to have a SNN of $2 \mathrm{~cm}$ in the fifth segment of the liver, which had not been observed on previous ultrasound (US). On the basis of the histological findings, we discuss the various hypotheses regarding the histogenesis of this rare lesion, which is still debated. Furthermore, this is the first case of laparoscopic resection of this uncommon benign liver lesion. 


\section{Case Report}

A 58-year-old woman was admitted to our ward following a history of several episodes of cramp-like pains in the right hypochondria irradiating to the ipsilateral scapula in the previous six months. On admission, routine laboratory data were normal. US of the upper abdomen showed multiple stones in the gallbladder without any lesion of the liver.

The patient underwent laparoscopic cholecystectomy for her gallstones. During the laparoscopic exploration, a single round lesion of $2 \mathrm{~cm}$ located on the edge of the fifth liver segment, about $2 \mathrm{~cm}$ from the bottom of the gallbladder, was found. No signs of extrahepatic primitive malignant lesions were observed.

Cholecystectomy and a wedge resection of the liver comprising the lesion were performed. The macroscopic finding of the resected specimen showed the presence of a lobular-shaped formation with a hard consistency. Extemporary histological examination performed on the liver mass showed a picture of tissue necrosis alone. No signs of tumor-like cells were noted. The patient had an uneventful recovery and was discharged on postoperative day 3. The definitive histological examination on hematoxylineosin stained sections revealed a nodule with a completely necrotic core, combined with eosinophil granular matter, calcifications, cholesterol, foam cells, and some inflammatory cells. It was delimited by a fibrotic capsule and by granulomatous tissue (fig. 1a, b). The diagnosis of SNN was made. Shikata's orcein staining showed the presence of elastic fibers in the capsule, but not in the central necrotic core (fig. 1c). On the other hand, the central necrotic core stained deeply for Gomori's silver stain, showing the collapse of the reticular fibers (fig. 1d). Ziehl-Neelsen, Gram and PAS stains did not reveal bacteria or fungi.

After surgery, plasma levels of some tumor markers such as CEA, CA 19-9 and alpha-fetoprotein were normal. Systemic examinations were performed to investigate the possibility of metastasis from the digestive tract or gynecological malignancy, but no abnormal findings were found on CT study. Clinical and instrumental controls performed up to 1 year after surgery showed no signs of disease or recurrence, and the patient is in good health.

\section{Discussion}

SNN of the liver is a rare benign lesion; to date fewer than 60 cases have been reported in the literature. Koea et al. reported one case taken from 500 referrals with liver masses presenting over a 2-year period [2], Berry [3] reported 2 lesions in 1,000 post mortems and Tsui et al. [4] documented 7 lesions in 4,000 patients. Most of the reported cases have been in males and the majority have been seen in the seventh and eighth decades of life. However, a number of cases have been found in patients in their thirties and forties [5-8], whereas Tsui et al. reported a case of a patient as young as 27 years old [4]. SNN, despite its name, may also be multiple [8].

Over three-quarters of these rare benign lesions occurred in the right lobe of the liver. In the initial description by Shepherd and Lee [1], all SNNs were reported on the surface of the liver and most of them were located on the right side, probably because the right lobe of the liver is easy to examine, but also because of the possibility that trauma may be involved in the pathogenesis of these lesions. Only four of the reported SNNs were found in the left lobe $[1,4]$. In a review of 21 cases performed by Imura et al., it was noted that in fifteen cases the tumor was $\leq 2.0 \mathrm{~cm}$ in diameter and that all the lesions were $<5.0 \mathrm{~cm}$; only in one case did the authors find a mass $8.5 \mathrm{~cm}$ in diameter [9]. In the literature, there is a reported association with tumor pathology in $>50 \%$ of cases [10].

This kind of lesion is normally accidentally diagnosed during a workup for other pathologies or is an occasional finding during abdominal surgery or autopsy. In the majority of cases, this condition is clinically silent, and often the patients complain of nonspecific and unclear symptoms. SNNs are usually first detected at US examination. Some features, such as lobular shape, well-delineated margin, and close proximity to the 
hepatic inflow structures, are suggestive of SNN, but are nondiagnostic [2]. Since conventional color/power Doppler imaging is insensitive to the slow flow of deep-seated blood vessels, the lack of blood flow signal cannot confirm a total absence of blood perfusion within the lesion. SNN usually appears as a well-defined lesion with no enhancement on contrast CT or MRI [11] or US [12], while some SNNs may have subtle peripheral enhancement on contrast MRI [13].

Often the radiological findings of SNN of the liver are similar to those of hepatic metastasis or other liver masses, such as pseudotumor, lymphoma, regenerative and dysplastic nodules, or some infectious processes [14]. Due to these similar radiological findings, making a correct differential diagnosis is often very difficult or impossible [13]. The intraoperative US findings of the lesion also simulate hepatic metastasis, even though lesion multiplicity had been elucidated [13]. Furthermore, the cytological evaluation of specimens obtained by needle biopsy is not useful to correctly distinguish between these nodules and hepatic metastasis, because it often reveals only the presence of necrotic tissue. Due to these difficulties, performing a liver biopsy in order to clarify the diagnosis may be mandatory.

From a pathological point of view, SNN appears well-delineated by a dense fibrohyalinized fibrotic capsule with a principally necrotic core containing an eosinophil granular matter combined with calcifications, cholesterol, foam cells, and some inflammatory cells. Characteristically Ziehl-Neelsen, Gram and PAS stains do not reveal bacteria or fungi, and the pathogenesis of these cases remains uncertain. The histological findings of our case were in keeping with the above-mentioned features.

The etiology of these rare benign lesions is still uncertain; at least three different hypotheses have been suggested as to their origin. Some authors argue that these nodules have a traumatic etiology or that they are the result of previous parasitic infestation $[1,4$, 5]. Others suggest that because of the finding of a 'feeding vessel' and the absence of a cystic space or other signs of parasitic infestation, the lesions could be the result of the sclerotic evolution of small hemangiomas $[2,3,8]$. Finally, the possibility that a SNN of the liver may develop by two or more different mechanisms cannot be denied [11]. We agree with this last hypothesis that at least two different pathogenetic mechanisms could be involved. To support this claim, we would like to show the histological differences between the case we report in this work and a previously observed case of ours (unpublished). In the latter, large feeding vessels were evident at the periphery of the lesion (fig. 2a). Furthermore, the central core was more fibrous than necrotic and Shikata's orcein staining, evidencing several circular-running elastic fibers, suggested the presence of sclerotic vessels (fig. 2b) with lumina occluded or reduced to a thin fissure, evidenced only by performing anti-CD31, anti-CD34 and anti-factor VIII immunostaining to highlight the presence of the endothelial cells (fig. 2c). Finally, Gomori's silver staining did not show the collapse of the reticular network, but evidenced some reticular fibers around the vascular lumina (fig. $2 \mathrm{~d}$ ). Consequently, we think that careful observation and special staining could be useful to highlight morphological differences in SNN, which suggest the hypothesis of a different histogenetic origin.

To our knowledge, the natural history of SNN is still unclear. De Luca et al. in their report observed a reduction in size of SNN during follow-up [10], whereas Imura et al. reported a case with rapid enlargement in diameter from $3 \mathrm{~cm}$ to $>8 \mathrm{~cm}$ in the space of seven months [9].

In conclusion, although the benign behavior of this lesion and the absence of symptomatology suggest that surgical resection is unnecessary, the radiological suspicion 


\begin{tabular}{r|l|l|l}
$\begin{array}{r}\text { Case Reports in } \\
\text { Gastroenterology }\end{array}$ & $\begin{array}{l}\text { Case Rep Gastroenterol 2008;2:149-154 } \\
\text { D0I: 10.1159/000128168 }\end{array}$ & Published online: April 24, 2008 & $\begin{array}{l}\text { O 2008 S. Karger AG, Basel } \\
\text { ISSN 1662-0631 } \\
\text { www.karger.com/crg }\end{array}$ \\
\hline
\end{tabular}

of SNN demands to perform a liver biopsy, and in case of failure, given the high association of SNN with malignant lesions, confirms resection as the treatment of choice in the management of this disease.

Fig. 1. a Nodule with a completely necrotic core, with calcifications, delimited by a fibrotic capsule. HE staining, overall magnification $50 \times$. b At higher power, granulomatous tissue is evident between the fibrous capsule and the necrotic tissue, consisting of eosinophil granular matter, calcifications, cholesterol, foam cells, and some inflammatory cells. HE staining, overall magnification $200 \times$. c Presence of elastic fibers in the capsule, but not in the central necrotic core. Shikata's orcein staining, overall magnification $100 \times$. d Central necrotic core showing collapse of reticular fibers. Gomori's silver staining, overall magnification $100 \times$.

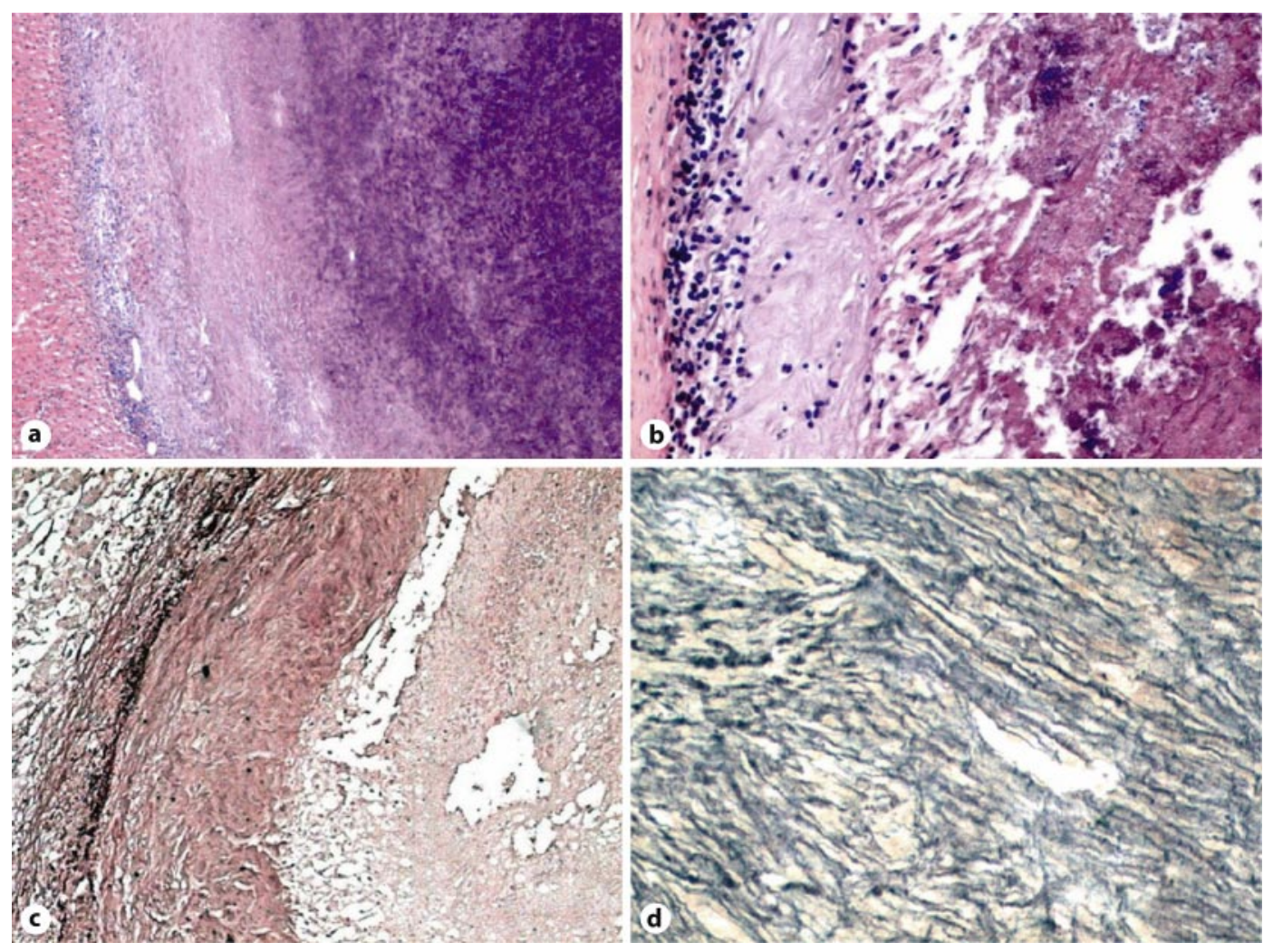




\begin{tabular}{r|l|l|l}
$\begin{array}{r}\text { Case Reports in } \\
\text { Gastroenterology }\end{array}$ & $\begin{array}{l}\text { Case Rep Gastroenterol 2008;2:149-154 } \\
\text { D0I: 10.1159/000128168 }\end{array}$ & Published online: April 24, 2008 & $\begin{array}{l}\text { O 2008 S. Karger AG, Basel } \\
\text { ISSN 1662-0631 } \\
\text { www.karger.com/crg }\end{array}$ \\
\hline
\end{tabular}

Fig. 2. a Large feeding vessels at the periphery of the lesion. The central core was more fibrous than necrotic. HE staining, overall magnification $50 \times$. b Circularly running elastic fibers suggesting the presence of sclerotic vessels. Shikata's orcein staining, overall magnification 100×. c CD34

immunostaining evidencing small vascular lumina. Overall magnification $200 \times$. d Gomori's silver staining evidenced some reticular fibers around the vascular lumina. No collapse of the reticular network. Overall magnification $100 \times$.

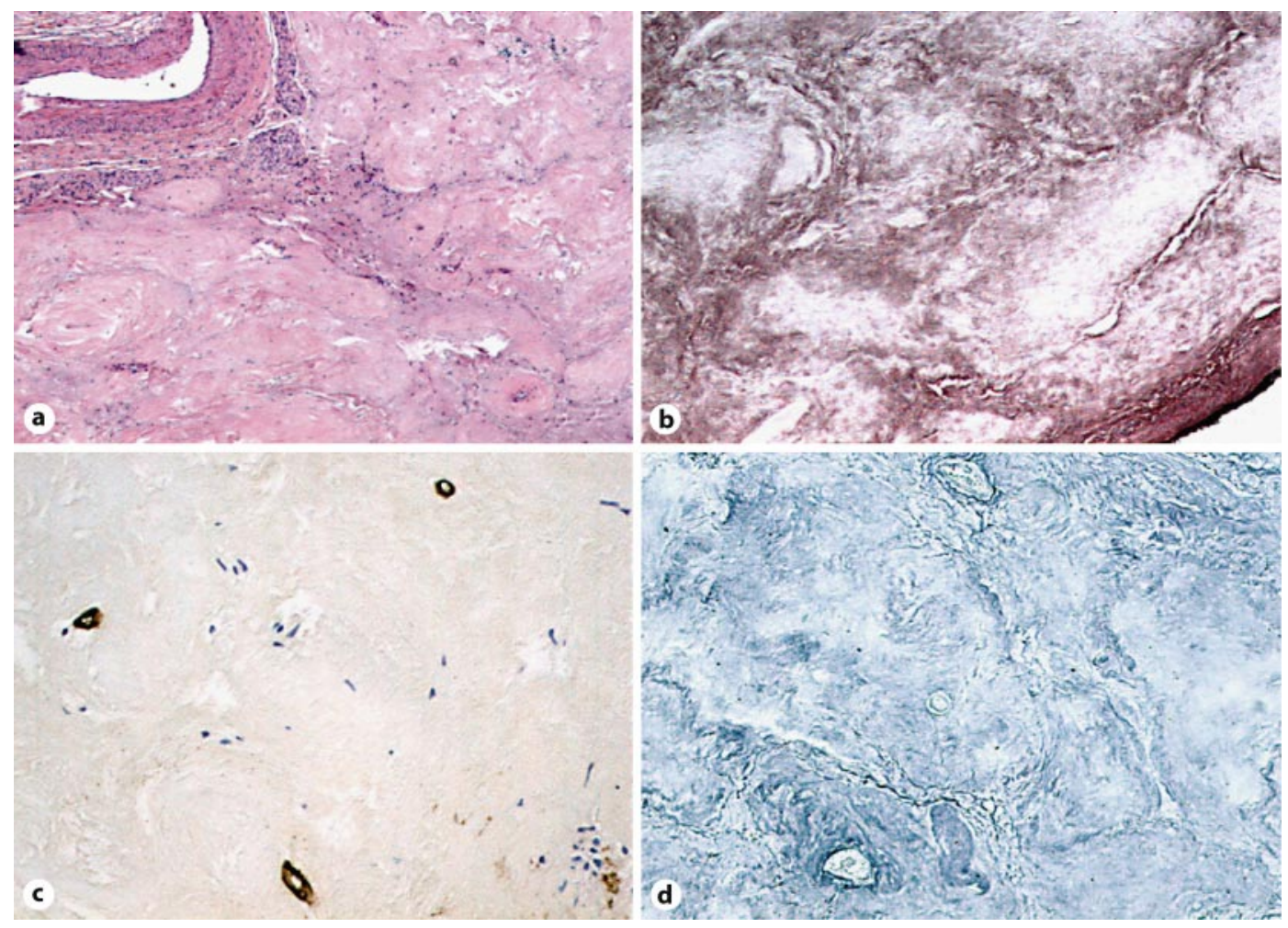




\section{References}

1 Shepherd NA, Lee G: Solitary necrotic nodules of the liver simulating hepatic metastases. J Clin Pathol 1983;36:1181-1183.

2 Koea J, Taylor G, Miller M, Rodgers M, McCall J: Solitary necrotic nodule of the liver: a riddle that is difficult to answer. J Gastrointest Surg 2003;7:627-630.

3 Berry CL: Solitary 'necrotic nodule' of the liver: a probable pathogenesis. J Clin Pathol 1985;38:1278-1280.

4 Tsui WM, Yuen RW, Chow LT, Tse CC: Solitary necrotic nodule of the liver: parasitic origin? J Clin Pathol 1992;45:975-978.

-5 Shepherd NA: Solitary necrotic nodule. J Clin Pathol 1990;43:348-349.

-6 Carella R, Fortunato C, Gubinelli M, D'Errico A, Mancini AM: Solitary necrotic nodule of the liver simulating a metastasis (in Italian). Pathologica 1993;85:573577.

7 Clouston AD, Walker NI, Prociv P: Parasitic origin of a solitary necrotic nodule of the liver. J Clin Pathol 1993;46:578.

8 Sundaresan M, Lyons B, Akosa AB: 'Solitary' necrotic nodules of the liver: an aetiology reaffirmed. Gut 1991;32:1378-1380.

-9 Imura S, Miyake K, Ikemoto T, Morine Y, Fujii M, Sano N, Shimada M: Rapidgrowing solitary necrotic nodule of the liver. J Med Invest 2006;53:325-329.

- 10 De Luca M, Luigi B, Formisano C, Formato A, De Werra C, Cappuccio M, Loffredo A, Forestieri P: Solitary necrotic nodule of the liver misinterpreted as malignant lesion: considerations on two cases. J Surg Oncol 2000;74:219-222.

-11 Iwase K, Higaki J, Yoon HE, Mikata S, Miyazaki M, Torikai K, Shirai Y, Awai K, Imakita M, Kamiike W: Solitary necrotic nodule of the liver. J Hepatobiliary Pancreat Surg 2002;9:120-124.

12 Wang Y, Yu X, Tang J, Li H, Liu L, Gao Y: Solitary necrotic nodule of the liver: Contrast-enhanced sonography. J Clin Ultrasound 2007;35:177-181.

13 Yoon KH, Yun KJ, Lee JM, Kim CG: Solitary necrotic nodules of the liver mimicking hepatic metastasis: report of two cases. Korean J Radiol 2000;1:165168.

14 Colagrande S, Politi LS, Messerini L, Mascalchi M, Villari N: Solitary necrotic nodule of the liver: imaging and correlation with pathologic features. Abdom Imaging 2003;28:41-44. 